\title{
Over-expression of cyclo-oxygenase-2 predicts poor survival of patients with nasopharyngeal carcinoma: a meta-analysis
}

- C-C Sim ${ }^{(a 1)}$ and E U-H Sim ${ }^{(a 1)}$

- DOI: https://doi.org/10.1017/S0022215120000614

- Published online by Cambridge University Press: 16 March 2020

\begin{abstract}
Objectives

The conclusive prognostic significance of cyclo-oxygenase- 2 has been determined in various cancers but not in nasopharyngeal carcinoma. Therefore, this study aimed to evaluate the relationship of cyclo-oxygenase-2 expression with the survival outcome and treatment response of nasopharyngeal carcinoma patients via a systematic meta-analysis approach.

Methods

A meta-analysis was conducted in compliance with the Preferred Reporting Items for Systematic Reviews and Meta-Analyses ('PRISMA') checklist. The primary clinical characteristics of patients, and hazard ratios with 95 per cent confidence intervals of overall survival data, were tabulated from eligible studies. The relationship of cyclo-oxygenase-2 expression with survival outcome (expressed as hazard ratio) and treatment response (expressed as odds ratio) in nasopharyngeal carcinoma patients was analysed, and explained with the aid of forest plot charts.
\end{abstract}

\section{Results and conclusion}

The pooled hazard ratio for overall survival was 2.02 (95 per cent confidence interval $=1.65-2.47$ ). This indicates that the over-expression of cyclo-oxygenase-2 is significantly associated with the poor survival of nasopharyngeal carcinoma patients. The pooled odds ratio of 0.98 (95 per cent confidence interval $=0.27-3.49$ ) reveals that over-expression of cyclo-oxygenase- 2 was not significantly related to the treatment outcome. 